\title{
High Susceptibility of Atherosclerotic Coronary Arteries to the Onset of Vasospasm and Angina Pectoris-like Symptoms due to Coronary Spasm in WHHLMI Rabbits
}

\author{
Tomonari Koike $^{1^{*}}$, Tatsuro Ishida ${ }^{2}$, Shiori Tamura ${ }^{3}$, Nobue Kuniyoshi ${ }^{1}$, Ying Yu ${ }^{1}$, Satoshi Yamada ${ }^{1}$, Ken-ichi Hirata ${ }^{2}$ and Masashi Shiomi, \\ ${ }^{1}$ Institute for Experimental Animals, Kobe University Graduate School of Medicine, Kobe, Japan \\ ${ }^{2}$ Division of Cardiovascular Medicine, Kobe University Graduate School of Medicine, Kobe, Japan
}

${ }^{3}$ Division of Comparative Pathophysiology, Kobe University Graduate School of Medicine, Kobe, Japan

\begin{abstract}
Objectives: We examined the relationship between atherosclerosis and provocation of coronary spasm as well as influence of coronary spasm on the onset of acute ischemic myocardial disease.

Methods and Results: Coronary spasm was provoked in anesthetized normal Japanese white (JW) rabbits and WHHLMI rabbits, an animal model for coronary atherosclerosis and myocardial infarction, by injecting ergonovine during the infusion of norepinephrine through a marginal ear vein. A decrease in contrast flow in the left circumflex artery was observed on coronary angiograms. Ischemic changes were observed on the electrocardiogram of $29 \%$ (2/7) of JW and $79 \%(27 / 34)$ of WHHLMI rabbits. The frequency of coronary spasm was significantly high in rabbits with severe coronary plaques showing diffuse lesions. In addition, the degree of contraction of coronary strips with atherosclerotic plaques was higher than that of normal coronary strips excised from JW rabbits stimulated by a combination of ergonovine and norepinephrine. Left ventricle motility in these vasospasm-positive rabbits, which was evaluated with echocardiograms was decreased by $29 \%$ following the ergonovine injection $(P<0.001)$, and every serum ischemic marker examined markedly increased 4 hours after the provocation of vasospasm.
\end{abstract}

Conclusions: The results of the present study demonstrated that atherosclerotic coronary arteries were positively related to the provocation of vasospasm, and vasospasm in severe atherosclerotic coronary segments evoked angina pectoris and/or non-fatal myocardial infarction.

Keywords: Angina pectoris; Atherosclerosis; Aoronary spasm; Ischemic myocardial injury; Ventricular dysfunction; WHHLMI rabbits

\section{Introduction}

The impact of coronary spasm on the onset of acute ischemic coronary events remains controversial. Several previous studies argued that coronary spasm was directly related to sudden cardiac death [14]. However, others recently indicated that the role of vasospasm in myocardial infarction remains unclear $[5,6]$. Thus, the relationship between coronary spasm and acute ischemic coronary events as well as that coronary spasm and the degree of atherosclerotic lesions have not been elucidated in detail. Previous studies demonstrated that the degree of coronary plaques on angiography was a predictor of vasospasm [7] and intravascular ultrasound studies revealed that atherosclerosis was present at sites with coronary spasm even in the absence of angiographically significant coronary artery disease [8]. However, vasospasm was also shown to occur at the site of angiographically normal coronary segments or in the presence of a nonobstractive atherosclerotic plaque [9]. Since difficulties are associated with determining the relationship between coronary plaques and vasospasm as well as that between coronary spasm and angina pectoris in clinical studies because of ethical problems, suitable animal models needed to be developed to more clearly understand coronary spasm and angina pectoris.

Several animals have been used as models in the study of coronary spasm. In ex vivo examinations, aortas and coronary arteries exposed to endogenous hyperlipidemia appeared to be hyper reactive to ergonovine [10]. Constrictive responses induced by constrictors were significantly augmented in a swine model fed a cholesterol diet, as was denudation of endothelium [11-13]. Although these findings contributed to clarifying the mechanisms involved in the provocation of vasospasm, the denudation of arterial endothelial layers does not occur physiological condition. Previous studies [13-15] examined the relationship between coronary spasm and the onset of acute coronary syndromes, but they did not use clinical findings. Lanza et al [16] reported that the cause of and mechanism responsible for coronary spasm were still poorly defined 60 years after the first report of variant angina despite of recent advances.

We developed a myocardial infarction-prone strain of the Watanabe heritable hyperlipidemic (WHHLMI) rabbit $[17,18]$ by selectively breeding the WHHL rabbit $[18,19]$, which has hypercholesterolemia because of a genetic defect in low-density lipoprotein receptors. We previously provoked coronary spasm in WHHLMI rabbits and demonstrated the associations between of coronary spasm and plaque disruption as well as ischemic myocardial damage [20]. However, we did not sufficiently examine the effects of coronary plaques on the provocation of coronary spasm or development of angina pectoris-

*Corresponding author: Tomonari Koike, Institute for Experimental Animals, Kobe University Graduate School of Medicine,7-5-1, Kusunoki-cho, Chuo-ku, Kobe 6500017, Japan, Tel: (+81) 78382 6901; E-mail: koiket@med.kobe-u.ac.jp

Received: December 15, 2014; Accepted: January 19, 2015; Published: January 24, 2015

Citation: Koike T, Ishida T, Tamura S, Kuniyoshi N, Yu Y, et al. (2015) High Susceptibility of Atherosclerotic Coronary Arteries to the Onset of Vasospasm and Angina Pectorislike Symptoms due to Coronary Spasm in WHHLMI Rabbits. Cardiol Pharmacol 4: 131 doi:10.4172/2329-6607.1000131

Copyright: $\odot 2015$ Koike T et al. This is an open-access article distributed under the terms of the Creative Commons Attribution License, which permits unrestricted use, distribution, and reproduction in any medium, provided the original author and source are credited. 
like symptoms, e.g. ventricular dysfunction and ischemic injury of myocardium.

We here in reanalyzed the data of our study [20] with the addition of data from four extra rabbits and ex vivo coronary contraction experiments to determine whether the severity of coronary plaques affected provocation of coronary spasm and acute ischemic myocardial events in WHHLMI rabbits.

\section{Methods}

\section{Animals}

We used 34 WHHLMI rabbits aged 12-29 months in experiments on the provocation of coronary spasm, and 15 male WHHLMI rabbits aged 8-15 months in the coronary contraction test. WHHLMI rabbits were bred at the Kobe University Graduate School of Medicine. As a control, 7 male Japanese White (JW) rabbits (Kitayama Labes, Co. Ltd., Ina, Japan) aged 8 months were used in the coronary spasm provocation test and examination of left ventricular function, while 15 age-matched male JW rabbits were used in the coronary contraction test. Rabbits resided individually in metal cages $(550 \mathrm{~mm}$ wide, $600 \mathrm{~mm}$ deep and $450 \mathrm{~mm}$ high) with a flat floor, and consumed standard rabbit chow (LRC4, Oriental Yeast Co., Ltd., Tokyo, Japan) at 120 g/day and water ad libitum. The animal rooms were maintained under a constant temperature $\left(22 \pm 2^{\circ} \mathrm{C}\right)$, relative humidity $(50-60 \%)$, ventilation rate (15 cycles/hour), and lighting cycle (12 hours light/dark). This study was approved by the Kobe University Animal Care and Use Committee (approval numbers: P080606, P091101), and animal experiments were conducted in accordance with the Regulations for Animal Experimentation of Kobe University, and Japanese regulations, such as the Act on Welfare and Management of Animals (Law No. 105; 1973, revised 2006), Standards Relating to the Care and Management of Laboratory Animals and Relief of Pain (Notification No. 88, 2006), and Fundamental Guidelines for Proper Conduct of Animal Experiment and Related Activities in Academic Research Institutions (Notice No.71, 2006).

\section{Anesthesia and euthanasia}

Rabbits were anesthetized with an intravenous injection of ketamine hydrochloride (15 mg/kg, Daiichi-Sankyo Co. Ltd., Tokyo, Japan) plus midazolam (1 mg/kg, Dormicum, Astellas Pharma Inc., Tokyo, Japan), and anesthesia was continued by the infusion of ketamine hydrochloride at $60 \mathrm{mg} / \mathrm{kg} / \mathrm{h}$. During experiments, oxygen was supplied through a face mask $(2.0 \mathrm{~L} / \mathrm{min}$ for rabbits $)$, and rabbits were warmed with a heating pad. Rabbits were euthanized with exsanguination under the intravenous administration of sodium pentobarbital $(30 \mathrm{mg} / \mathrm{kg})$.

\section{Isometric tension measurement of coronary arterial strips}

The Left Anterior Descending artery (LAD) and Left Circumflex Arteries (LCX) were immediately isolated after euthanasia, cleaned of surrounding tissue, and cut into 2-mm-wide and 15 -mm-long helical strips. The endothelium was denuded by rubbing the vessels with wet filter paper. Isometric tension was measured in the coronary strips as previously described $[10,21]$. Briefly, coronary strips were suspended in organ baths containing Krebs buffer, and an initial preload of $0.5 \mathrm{~g}$ was applied. After 2 hours for equilibration, test contractions were induced by adding $20 \mathrm{mmol} / \mathrm{L} \mathrm{KCl}$. When the developed tension attained its peak value, strips were relaxed by rinsing with buffer. Constriction responses were evaluated as the percentage of maximum tension induced by 20 $\mathrm{mmol} / \mathrm{L} \mathrm{KCl}$. Concentration-response relationships were determined by the cumulative addition of norepinephrine and ergonovine (all from Sigma-Aldrich, St. Louis, MO). Furthermore, coronary strips were stimulated with cumulative doses of ergonovine after the tone had been moderately increased by a threshold concentration $\left(\sim \mathrm{EC}_{20}\right)$ of norepinephrine $(1 \mu \mathrm{M})$.

\section{Provocation of coronary spasm}

The provocation and evaluation of coronary spasm were performed as previously described [20], and the study design was shown in Figure 1.

Evaluation of ventricular contractile dysfunction and myocardial ischemia

Echocardiograms were performed using 13 WHHLMI rabbits and 7 JW rabbits with a Philips Envisor C echocardiograph (Philips Inc., Eindhoven and the Netherlands) [20]. Left ventricular function was evaluated by fractional shortening, which was calculated as 1 Systolic Left Ventricular Diameter (LVDs)/Diastolic Left Ventricular Diameter (LVDd). Serum biomarkers for ischemic myocardial damage (heart-type fatty acid-binding protein [H-FABP], cardiac troponin-I [cTroponin-I], and myoglobin) were assayed with ELISA kits (Life Diagnostics Inc., West Chester, PA, USA) before the injection of vasoconstrictors, and 4 hours after ischemic changes occurred on ECG using 20 WHHLMI rabbits.

\section{Preparation of coronary sections}

Rabbits were euthanized after the examination. Hearts were excised and immersion-fixed with a $10 \%$ neutral buffered formalin solution, and embedded in paraffin. Coronary arterial segments were prepared as reported previously [22]. Sections were stained with elastic van Gieson stain. Coronary stenosis was evaluated as cross-sectional narrowing (\%) calculated by dividing the lumen area by the area surrounded with an internal elastic lamina.

\section{Assay of serum lipid levels}

Serum total cholesterol and triglyceride levels were assayed enzymatically with kits at 12 months of age using sera obtained after 15 hours of fasting.

\section{Statistical analysis}

Data are presented as the mean \pm the Standard Error of the Mean (SEM). Statistical analyses were performed for mean values with the signed Wilcoxon test, Mann-Whitney U-test, or Student's $t$-test; and for frequency with the chi-square test. To compare of mean values among multiple groups, we performed the Bonferroni test. A value of $\mathrm{P}<0.05$ was taken as significant.

\section{Results}

\section{Baseline data of WHHLMI rabbits}

As shown in Table 1, all WHHLMI rabbits had hypercholesterolemia and each coronary artery had atherosclerotic plaques. Coronary stenosis (evaluated as cross-sectional narrowing) of more than $75 \%$ was observed in 23 rabbits $(67.6 \%)$.

\section{Contractile responses in coronary helical strips}

Norepinephrine and ergonovine induced contractions in control JW and WHHLMI coronary strips in a dose-dependent manner (Figure 2). There is no significant difference between WHHLMI and JW rabbits in maximum tension induced by $20 \mathrm{mmol} / \mathrm{L} \mathrm{KCl}$ (data not shown) as 


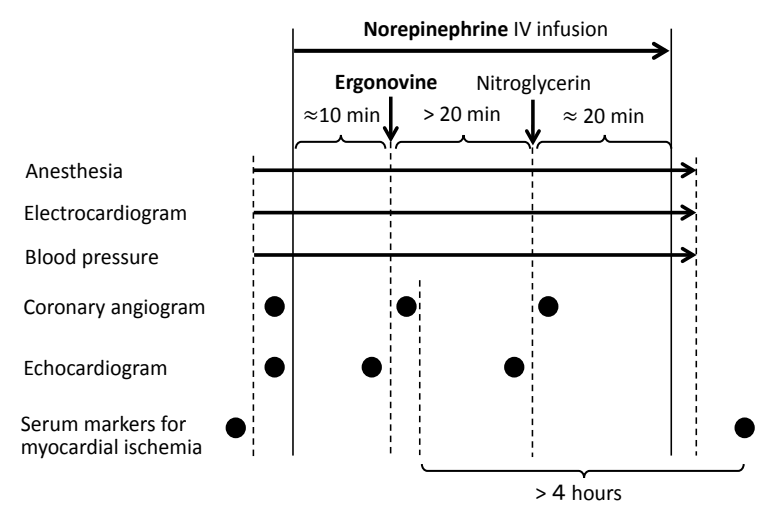

Figure 1: Study design for the provocation of coronary spasm. Coronary spasm was provoked with an intravenous injection of ergonovine maleate (Tokyo Chemical Industry Co., Ltd. Tokyo, Japan) at a dose of 0.45 $\mu \mathrm{mol} / \mathrm{kg}$ during the infusion of norepinephrine (Daiichi-Sankyo Co., Ltd., Tokyo, Japan) at a rate of $12 \mathrm{nmol} / \mathrm{kg} / \mathrm{min}$ through a marginal ear vein. Nitroglycerin (Hikari Pharmaceutical Co., Ltd., Tokyo, Japan) was injected intravenously $(10 \mu \mathrm{g} / \mathrm{kg}) 20-30$ minutes after the onset of ischemic changes on electrocardiogram (ECG) to reverse coronary spasm. The occurrence of coronary spasm was monitored with coronary angiograms using an X-ray apparatus (OPESCOPE PLENO; Shimadzu Corporation, Kyoto, Japan), and ECG were measured using an amplifier (AB-621G; Nihon Kohden, Tokyo, Japan) and PowerLab/8SP (ADInstruments Pty Ltd, Bella Vista, Australia), or an electrocardiograph (CARDIOSUNY a6000 AX-D, Fukuda M-E Kogyo, Co., Ltd., Tokyo, Japan) . Blood pressure was also monitored invasively at the femoral artery.

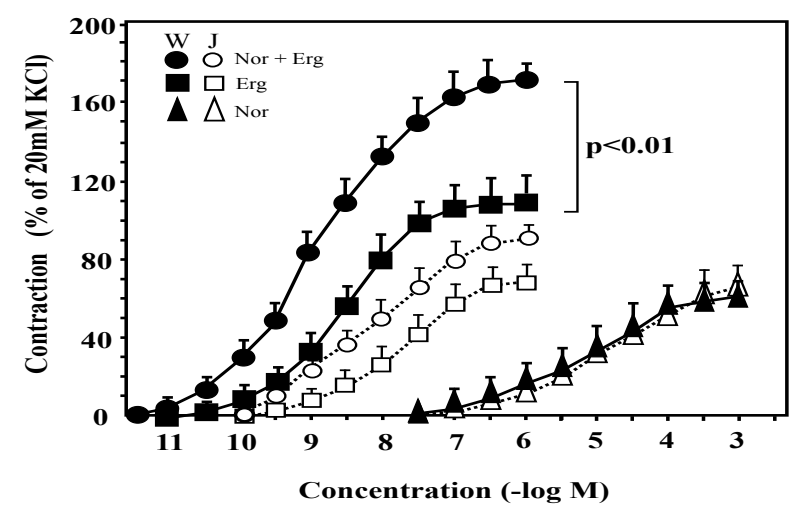

Figure 2: Contraction-response of the endothelium-denuded helical coronary strips of rabbits treated with norepinephrine and ergonovine. The abscissa shows the final concentration of the agonists, and the ordinate shows the percentage of maximal tension induced by $20 \mathrm{mmol} / \mathrm{L} \mathrm{KCL}$. Coronary strips were treated with norepinephrine (Nor, $\boldsymbol{\Delta}$ or $\Delta$ ), ergonovine (Erg, - or $\square$ ), and $1.0 \mu \mathrm{M}$ norepinephrine plus ergonovine (Nor + Erg, $\bullet$ or $\circ)$. Each point represents the mean $\pm \operatorname{SEM}(n=5)$. Statistical analyses were performed with the Student's $t$-test. Closed symbols $(\boldsymbol{\Lambda}, \bullet, \bullet)$ represent data from WHHLMI rabbits $(\mathrm{W})$ and open symbols $(\Delta, \square, \circ)$ represent data from normal JW rabbits $(\mathrm{J})$. Bars indicate the SEM.

similar to a previous study [9]. No significant differences were observed in the contraction by norepinephrine (triangles) between WHHLMI and control strips. The concentration-response curves for ergonovine after contractions with norepinephrine in WHHLMI strips (closed circles) exhibited a left and upward shift from the control (open circles). The $\mathrm{E}_{\max }$ for combined treatment was $174 \pm 15 \%$ in WHHLMI coronary strip, and was $89 \pm 8 \%$ in the normal coronary strips. These results indicated that atherosclerotic WHHLMI coronary strips were hypersensitive and hyper reactive to these agents relative to normal coronary strips.

\section{Occurrence of coronary spasm}

During the vasospasm provocation test, ECG showed ischemic changes in WHHLMI rabbits (Figure 3), e.g. ST depression/ elevation, T-wave elevation/inversion, poor $\mathrm{R}$-wave progression, deep Q-wave, and ventricular premature complex. The frequency of these abnormalities was markedly higher in WHHLMI rabbits (27/34, $79.4 \%, \mathrm{P}=0.007)$ than in normal JW rabbits $(2 / 7,28.6 \%)$. We confirmed development of coronary spasm with coronary angiography. Figure 4 shows the provocation of angiographical coronary spasm in WHHLMI rabbits in vivo. Contrast flow was markedly decreased after the injection of ergonovine (Figure 4A), and the area with decreased perfusion in LCX corresponded to segments with plaques (sections 7-16) (Figure $4 \mathrm{~B})$. Regarding the relationship between coronary plaques and the development of coronary spasm (Figure 4C), the frequency of coronary spasm was proportional to the degree of coronary stenosis $(\mathrm{P}=0.01)$ and the number of coronary segments with lesions $(\mathrm{P}=0.024)$. Since coronary stenosis with $>75 \%$ cross-sectional narrowing is often used as the level of significant stenosis in clinical analysis, we examined the relationship between significant stenosis and vasospasm provocation. The frequency of coronary segments with more than $75 \%$ stenosis was significantly high in the vasospasm-positive WHHLMI rabbits $(32.3$ $\pm 5.9 \%, \mathrm{P}=0.036)$ than in vasospasm-negative WHHLMI rabbits $(8.2$ $\pm 6.0 \%)$. These results demonstrated that atherosclerotic coronary arteries were highly susceptible to the provocation of vasospasm.

\section{Changes in the motility of the left ventricular wall}

Systolic blood pressure was significantly increased with the spasmogen treatments, but was significantly decreased at the end of the experiment $(96 \pm 6.2 \mathrm{mmHg}, \mathrm{P}=0.018)$ than the baseline $(131 \pm$ $4.6 \mathrm{mmHg}$ ). Since these results may suggest cardiac dysfunction due to coronary spasm, we further analyzed cardiac functions by echocardiogram. Table 2 shows the results of echocardiograms during the vasospasm provocation test. Although fractional shortening was already significantly lower at the baseline in WHHLMI rabbits $(32.5$ $\pm 1.2, \mathrm{P}=0.004)$ than in normal JW rabbits $(41.5 \pm 3.1)$, it was further decreased after the spasmogen treatments $(23.0 \pm 0.6, \mathrm{P}<0.001)$. These results indicated that coronary spasm lead to cardiac dysfunction in WHHLMI rabbits.

\section{Evaluation of ischemic damage to the left ventricular wall}

Table 3 shows changes in serum markers for ischemic myocardial injury in WHHLMI rabbits. These serum markers were within human normal ranges at the baseline. However, they were markedly increased after the provocation of vasospasm, which confirmed that coronary spasm induced ischemic injury in the myocardium.

\section{Discussion}

These results of the present study demonstrated that coronary spasm was induced frequently in WHHLMI rabbits with diffuse atherosclerotic plaques showing more than $75 \%$ coronary stenosis by pharmacological treatments, and coronary spasm caused cardiac dysfunction and ischemic injury to the myocardium. These results suggest that angina pectoris and/or non-fatal myocardial infarction developed due to the provocation of coronary spasm.

In the present study, the frequency of coronary spasm in WHHLMI rabbits with severe coronary stenosis and diffuse atherosclerotic plaques was significantly higher than that in WHHLMI rabbits with less stenosis and focal plaques in spite of in serum lipid levels being similar (data not shown). Previous studies reported a reduction in arterial 
A

Baseline

$\begin{array}{lllllllllll}\text { I } & \text { II } & \text { III } & \text { aVR } & \text { aVL } & \text { aVF } & \text { V-1 } & \text { V-2 } & \text { V-3 } & \text { V-4 } & \text { V- }\end{array}$

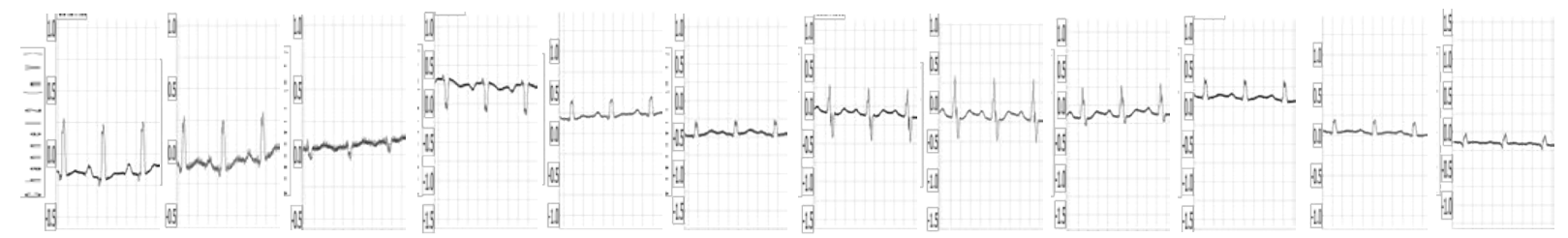

After the ergonovine injection
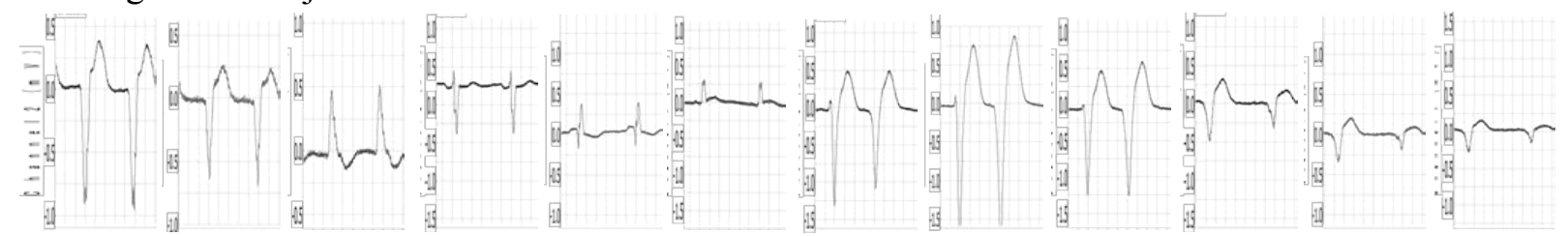

After stopping the norepinephrine infusion
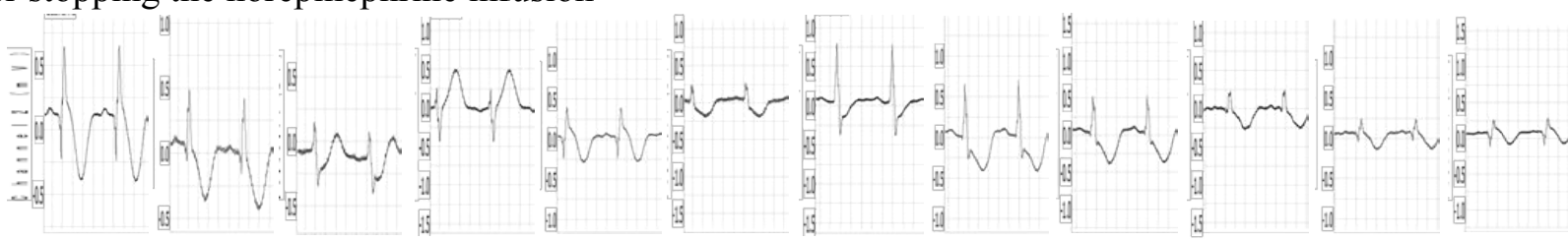

B

Examined rabbits

Any ischemic changes

ST segment depression

ST segment elevation

T-wave inversion

T-wave elevation

Poor R-wave progression

Q-wave

Ventricular premature complex

Left bundle branch block

\section{Rabbits}

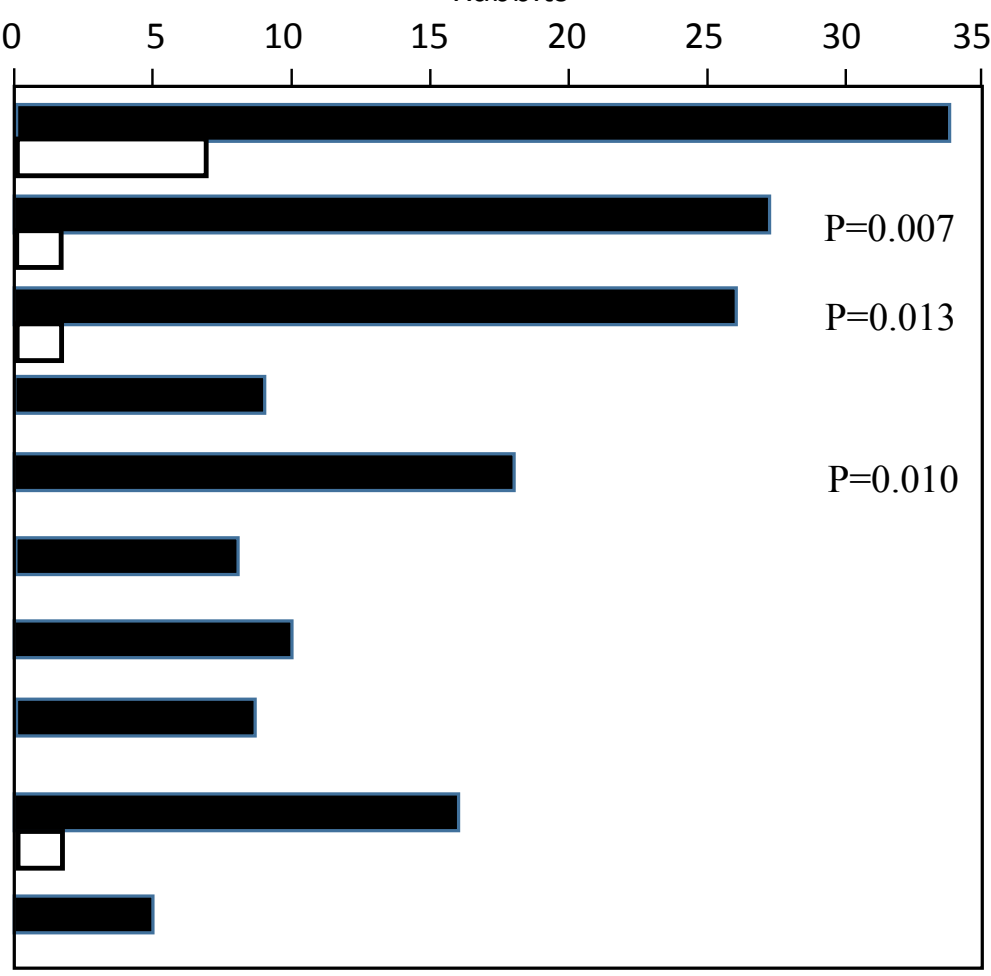

Figure 3: The electrocardiograms (ECG) of rabbits treated with ergonovine bolus injection under norepinephrine infusion. (A) Representative ECG changes in a WHHLMI rabbit during the experiments. (B) Frequency of ischemic patterns on ECG in normal rabbits ( $n=7$, open bars) and WHHLMI rabbits ( $n=34$, solid bars) treated with an ergonovine bolus injection under the infusion of norepinephrine. Statistical analyses were performed with the chi-square test. 


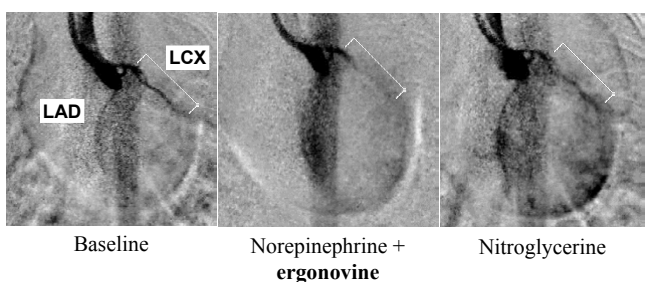

B

C

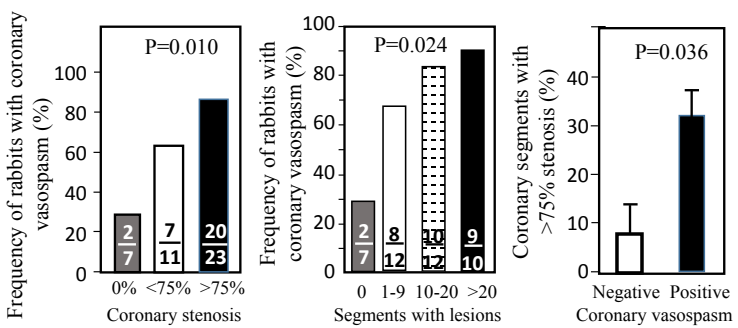

Figure 4: Relationship between coronary plaques and coronary spasm in WHHLMI rabbits. (A) Coronary angiograms at the baseline (left), after the ergonovine injection (center), and after a nitroglycerine injection (right). (B) Coronary stenosis evaluated as cross-sectional narrowing of a left circumflex artery as indicated on the angiogram. (C) Relationship between coronary plaques and the provocation of coronary spasm. Data are represented as the mean \pm SEM. Statistical tests were performed with the chi-square test for the frequency and with the Student's $t$-test for the mean value. LAD, left anterior descending artery, LCX, left circumflex artery.

\begin{tabular}{|c|c|}
\hline Examined rabbits & 34 \\
\hline Gender (female: male) & $11: 23$ \\
\hline Age (months) & $18.0 \pm 0.8$ \\
\hline Body weight (kg) & $3.32 \pm 0.07$ \\
\hline \multicolumn{2}{|c|}{ Serum lipid levels at 12 months old (mmol/L) } \\
\hline Total cholesterol & $21.9 \pm 0.8$ \\
\hline Triglyceride & $4.1 \pm 0.4$ \\
\hline \multicolumn{2}{|c|}{ Coronary plaques } \\
\hline Examined segments & $21.5 \pm 1.0$ \\
\hline Segments with lesions & $14.6 \pm 1.4$ \\
\hline $\begin{array}{l}\text { Animals with more than } 75 \% \text { coronary } \\
\text { stenosis }\end{array}$ & $67.6 \%(23 / 34)$ \\
\hline Segments with more than $75 \%$ stenosis & $9.7 \pm 1.6$ \\
\hline Maximum stenosis (\%) & $78.2 \pm 3.3$ \\
\hline \multicolumn{2}{|c|}{ Blood pressure at the femoral artery $(n=13, \mathrm{mmHg})$} \\
\hline Systolic & $131 \pm 4.6$ \\
\hline Diastolic & $68 \pm 3.5$ \\
\hline Heart rate (beats/min) & $46 \pm 6.0$ \\
\hline
\end{tabular}

Data are represented as the mean \pm SEM.

Table 1: Baseline data of WHHLMI rabbits provoked with coronary spasm

\begin{tabular}{|l|l|l|l|}
\hline & \multicolumn{2}{|l|}{ Left ventricular diameter $(\mathrm{mm})$} & $\begin{array}{l}\text { Fractional } \\
\text { Shortening }\end{array}$ \\
\hline & Diastole & Systole & $41.5 \pm 3.1$ \\
\hline Normal JW rabbits $(n=7)$ & $13.2 \pm 1.0$ & $7.8 \pm 0.8$ & 4 \\
\hline WHHLMI rabbits $(n=13)$ & & & \\
\hline Baseline & $14.5 \pm 1.0$ & $9.8 \pm 0.6$ & $32.5 \pm 1.2^{*}$ \\
\hline Norepinephrine infusion & $15.1 \pm 0.5$ & $10.6 \pm 0.4$ & $29.7 \pm 1.3$ \\
\hline Norepinephrine+Ergonovine & $15.1 \pm 0.7$ & $11.6 \pm 0.5 \#$ & $23.0 \pm 0.6 \#$ \\
\hline
\end{tabular}

Data are represented as the mean \pm SEM. Statistical analyses were performed with the Student's $t$-test for differences between normal and WHHLMI rabbits, and with the Bonferroni test for WHHLMI rabbits in the coronary vasospasm provocation test. *, $\mathrm{P}=0.004$ (JW vs WHHLMI baseline); $\#, \mathrm{P}=0.009$ (baseline vs combination treatment); \#, $\mathrm{P}<0.001$ (baseline vs combination treatment)

Table 2: Left ventricular wall motility in WHHLMI rabbits with coronary spasm

\begin{tabular}{|l|l|l|l|}
\hline & $\begin{array}{l}\mathrm{H}-\mathrm{FABP} \\
(\mathrm{ng} / \mathrm{ml})\end{array}$ & $\begin{array}{l}\text { cTroponin-I } \\
(\mathrm{ng} / \mathrm{ml})\end{array}$ & $\begin{array}{l}\text { Myoglobin } \\
(\mathrm{ng} / \mathrm{ml})\end{array}$ \\
\hline Baseline & $2.07 \pm 0.48$ & $0.018 \pm 0.018$ & $67.8 \pm 21.9$ \\
\hline $\begin{array}{l}4 \text { hours after the } \\
\text { treatments }\end{array}$ & $28.0 \pm 4.9$ & $2.71 \pm 0.83$ & $907 \pm 127$ \\
\hline P-value & $<0.001$ & $<0.001$ & $<0.001$ \\
\hline
\end{tabular}

Human normal ranges were $<6.2 \mathrm{ng} / \mathrm{ml}$ in H-FABP, $<0.04 \mathrm{ng} / \mathrm{ml}$ in cTroponin-I and $20-80 \mathrm{ng} / \mathrm{ml}$ in myoglobin. Data are presented as the mean \pm SEM. Statistical analyses were performed with the Wilcoxon signed rank test. H-FABP, heart type fatty acid binding protein; cTroponin-I, cardiac troponin-I

Table 3: Changes in serum markers for ischemic myocardial damage in WHHLMI rabbits with coronary spasm $(n=20)$

endothelial function in atherosclerotic arteries [23, 24]; therefore, the present results suggest that a dysfunction in the arterial endothelium of atherosclerotic plaques may affect the constriction of coronary arteries. In addition, ex vivo studies using endothelial-denudated coronary strips demonstrated the hypersensitivity and hyperreactivity of atherosclerotic strips. These results are consistent with previous findings [11,12,21]. Therefore, we speculated that the enhanced contractile response observed in atherosclerotic coronary strips may primarily be attributed to the hyperreactivity of medial smooth muscle cells. Maseri et al [25] demonstrated that the response to constrictor stimuli also extended to the adjacent segments and coronary spasm can be multi-focal and/or diffuse. Miyao et al [26] and Tsujita et al [27] also showed that coronary spasm was not a localized, but a generalized disorder that involved the entire coronary arteries and was associated with intimal thickening. These findings were consistent with the angiographical results in the present study; therefore, coronary spasm in WHHLMI rabbits is, from a pathophysiological aspect, relevant to that in humans.

After the provocation of coronary spasm, the left ventricle motility in rabbits with coronary spasm evaluated with echocardiograms was decreased, and serum markers for ischemic myocardial injury were markedly increased. These results suggested the onset of angina pectoris-like symptoms and/or non-fatal myocardial infarction, although the frequency of occlusive thrombi after the disruption of coronary plaques was very low [20]. Previous studies asserted that coronary spasm plays an important role in the pathogenesis of not only variant angina, but ischemic coronary disease, including other forms of angina, acute myocardial infarction, arrhythmias, and ischemic sudden death [28-31]. These findings were consistent with the results of the present study; therefore, we speculated that the WHHLMI rabbit is useful as an animal model of experimentally provoked coronary spasm and subsequent myocardial ischemia.

\section{Conclusion}

The present results suggest that atherosclerosis in coronary arteries 
Citation: Koike T, Ishida T, Tamura S, Kuniyoshi N, Yu Y, et al. (2015) High Susceptibility of Atherosclerotic Coronary Arteries to the Onset of Vasospasm and Angina Pectoris-like Symptoms due to Coronary Spasm in WHHLMI Rabbits. Cardiol Pharmacol 4: 131. doi:10.4172/2329-6607.1000131

Page 6 of 6

contributes to the development of coronary spasm, and coronary spasm in severe atherosclerotic lesions results in acute ischemic myocardial damage, which is similar to angina pectoris in humans. The WHHLMI rabbit is a good animal model for coronary spasm and related cardiac dysfunctions.

\section{Conflict of interesting}

Nothing to declare.

\section{Sources of Funding:}

This work was supported, in part, by a grant for Research on Biological Resources and Animal Models for Drug Development from the Ministry of Health, Labor, and Welfare of Japan, by Grants-inAid for scientific research from the Ministry of Education, Culture, Sports, and Technology, Japan 23300157), and by a research grant from Daiichi-Sankyo Co. Ltd., Tokyo, Japan,

\section{Acknowledgements:}

We would like to thank Dr. Norihisa Nitta, and Dr. Akinaga Sonoda, Shiga University of Medical Science, Japan, for coronary angiography on rabbits.

\section{References}

1. Figueras J, Cortadellas J, Barrabes J, Domingo E, Hermosilla E (2007) Longterm Cardiac Events in Men and Women with Angina at Rest and Transient ST Segment Elevation. Heart 93: 379-380.

2. Krahn AD, Healey JS, Chauhan V ,Simpson CS, Birnie DH, et al. (2009) Systematic Assessment of Patients with Unexplained Cardiac Arrest: Cardiac Arrest Survivors with Preserved Ejection Fraction Registry (CASPER). Circulation 120: 278-285.

3. Ong P, Athanasiadis A, Hill S, Vogelsberg H, Sechtem U, et al. (2008) Coronary Artery Spasm as a Frequent cause of Acute Coronary Syndrome. J Am Coll Cardiol 52: 523-527.

4. Ong P, Athanasiadis A, Borgulya G, Kaski JC, Sechtem U, et al. (2012) High Prevalence of a Pathological Response to Acetylcholine Testing in Patients with Stable Angina Pectoris and Unobstructed Coronary Arteries. J Am Coll Cardiol 59: 655-662.

5. Reynolds HR (2014). Mechanism of myocardial infarction without obstructive coronary artery disease. Trends Cardiovasc Med 24: 170-176.

6. Krahn AD, Healey JS, Chauhan V, Simoson CS, Champagne J, et al. (2009) Systematic assessment of patients with unexpected cardiac arrest: Cardiac arrest survivors with preserved ejection fraction registry (CASPER). Circulation 120: 278-285.

7. Harding MB, Leithe ME, Mark DB, Nelson CL, Hermiller JB, et al. (1992) Ergonovine maleate testing during cardiac catheterization: a 10-year perspective in 3,447 patients without significant coronary disease or Prinzmetal's variant angina. J Am Coll Cardiol 20: 107-111.

8. Koyama J, Yamagishi M, Tamai J, Kawano S, Daikoku S, et al. (1995) Comparison of vessel wall morphologic appearance a sites of focal and diffuse coronary vasospasm by intravascular ultrasound Am Heart J 130: 440-5.

9. Crea F, Liuzzo G (2013) Pathogenesis of acute coronary syndromes. J Am Coll Cardiol 61: 1-11.

10. Yokoyama M, Akita H, Mizutani T, FukuZaki H, Watanabe Y (1983) Hyperreactivity of coronary arterial smooth muscles in response to ergonovine from rabbits with hereditary hyperlipidemia. Circ Res 53: 63-71.

11. Shimokawa $H$, Tomoike $H$, Nabeyama S, Yamamoto H, Ishii $Y$, et al. (1985) Coronary artery spasm induced in miniature swine: Angiographic evidence and relation to coronary atherosclerosis. Am Heart J 110: 300-310.

12. Egashira K, Tomoike H, Yamamoto Y, Yamada A, Nakamura M, et al. (1986) Histamine-induced coronary spasm in regions of intimal thickening in miniature pigs: roles of serum cholesterol and spontaneous or induced intimal thickening Circulation 74: 826-837.

13. Kuga $T$, Tagawa $H$, Tomoike $H$, Nakamura M, Ohara $Y$, et al. (1993) Role of coronary artery spasm in progression of organic coronary stenosis and acute myocardial infarction in a swine model-importance of mode of onset and duration of coronary artery spasm. Circulation 87: 573-582.
14. Constantinides $P$, Whyman $J(1962)$ Infarction and infarctoid necrosis in atherosclerotic rabbits. J Atheroscler Res 2: 285-305.

15. Nakamura M, Abe S (1997) An Experimental Induction of Acute Myocardia Infarction and Arterial Thrombosis in Rabbits. Ann N Y Acad Sci 811: 424-428.

16. Lanza AG, Careri G, Crea F (2011) Mechanisms of coronary artery spasm Circulation 124: 1774-1782

17. Shiomi M, Ito T, Yamada S, Fan J, Kawashima S (2003) Development of an animal model for spontaneous myocardial infarction (WHHLMI rabbit). Arterioscler Thromb Vasc Biol 23: 1239-1244.

18. Shiomi M, Ito T (2009) The Watanabe heritable hyperlipidemic (WHHL) rabbit, its characteristics and history of development: a tribute to the late Dr. Yoshio Watanabe. Atherosclerosis 207: 1-7.

19. Watanabe $Y(1980)$ Serial inbreeding of rabbits with hereditary hyperlipidemia (WHHL-rabbit). Atherosclerosis 36: 261-268.

20. Shiomi M, Ishida T, Kobayashi T,Yamada S, koike T, et al. (2013) Vasospasm of Atherosclerotic Coronary Arteries Precipitates Acute Ischemic Myocardia Damage in Myocardial Infarction-Prone Strain of the Watanabe Heritable Hyperlipidemic Rabbits. Arterioscler Thromb Vasc Biol 33: 2518-2523.

21. Ishida T, Kawashima S, Hirata K, Sakoda T, Miwa Y, et al. (2001) Serotonininduced hypercontraction through 5-hydroxytryptamine $1 \mathrm{~B}$ receptors in atherosclerotic rabbit coronary arteries. Circulation 103: 1289-1295.

22. Shiomi M, Ito T, Shiraishi M, Watnabe $Y$ (1992) Inheritability of atherosclerosis and the role of lipoproteins as risk factors in the development of atherosclerosis in WHHL rabbits: Risk factors related to coronary atherosclerosis are different from those related to aortic atherosclerosis. Atherosclerosis 96: 43-52.

23. Hirata K, Akita H, Yokoyama M, Watnabe $Y$ (1992) Impaired vasodilatory response to atrial natriuretic peptide during atherosclerosis progression. Arterioscler Thromb 12: 99-105

24. Kanazawa K, Kawashima S, Mikami S, Miwa Y, Hirata K, et al. (1996) Endothelial constitutive nitric oxide synthase protein and mRNA increased in rabbit atherosclerotic aorta despite impaired endothelium-dependent vascular relaxation. Am J Pathol 148: 1949-1956.

25. Maseri A, Beltrame JF, Shimokawa H (2009) Role of Coronary vasoconstriction in ischemic heart disease and search for novel therapeutic targets. Circ J 73 394-403.

26. Miyao Y, Kugiyama K, Kawano H, Motoyama T, Ogawa H et al. (2000) Diffuse intimal thickening of coronary arteries in patients with coronary spastic angina. J Am Coll Cardiol 36: 432-437.

27. Tsujita K, Sakamoto K, Kojima S, Takaoka N, Tayama S, et al. (2013) Coronary plaque component in patients with vasospastic angina: $A$ virtual histology intravascular ultrasound study. Int J Cardiol 168: 2411-2415.

28. Shimokawa H (2000) Cellular and molecular mechanisms of coronary artery spasm. Lessons from animal models. Jpn Circ J 64: 1-12.

29. Wang CH, Kuo LT, Hung MJ, Cherng WJ (2002) Coronary vasospasm as a possible cause of elevated cardiac troponin I in patients with acute coronary syndrome and insignificant coronary artery disease. Am Heart J 144: 275-281.

30. MacAlpin RN (1993) Cardiac arrest and sudden unexpected death in variant angina: complications of coronary spasm that can occur in the absence of severe organic coronary stenosis. Am Heart J 125: 1011-1017.

31. Togashi I, Sato T, Soejima K, Miyoshi S Ogawa S et al. (2013) Sudden cardiac arrest and syncope triggered by coronary spasm. Int J Cardiol 163: 56-60. 